\title{
Elliptic model for space-time correlations in turbulent shear flows
}

\author{
Guo-Wei He $\mathrm{He}^{1,2, *}$ and Jin-Bai Zhang ${ }^{1}$ \\ ${ }^{1}$ LNM, Institute of Mechanics, Chinese Academy of Sciences, Beijing, 100080, People's Republic of China \\ ${ }^{2}$ Department of Aerospace Engineering, Iowa State University, Ames, Iowa 50011-2271, USA
}

(Received 6 March 2006; published 24 May 2006)

\begin{abstract}
An elliptic model for space-time correlations in turbulent shear flows is proposed based on a second order approximation to the iso-correlation contours, while Taylor's hypothesis implies a first-order approximation. It is shown that the space-time correlations are mainly determined by their space correlations and the convection and sweeping velocities. This model accommodates two extreme cases: Taylor's hypothesis at vanishing sweeping velocity and the sweeping hypothesis at vanishing convection velocity. The result is supported by the data from the direct numerical simulation of turbulent channel flows.
\end{abstract}

DOI: 10.1103/PhysRevE.73.055303

PACS number(s): 47.27.Ak, 05.45.-a, 47.27.E-, 47.27.N-

The correlation of velocities at two time and two points, or simply space-time correlation, is a fundamental description on space-time fluctuations in turbulent flows. It provides more information on spatial-temporal structures than space correlation alone [1]. However, there is little research on space-time correlations [2-9], while most of previous research is devoted to space correlations [10]. In isotropic turbulence, Kraichnan [2] proposed the sweeping hypothesis in which the space-time correlations are mainly determined by space correlations and a sweeping velocity, leading to a universal form of space-time spectra. However, in turbulent shear flows, shear introduces new scales and adds more complexity to the space-time correlations. In the case of weak shear rates, Lesile [11] used the perturbation method to calculate the space-time correlations; Gotoh and Kaneda [12] used the Taylor series expansions to calculate the space-time correlations for short time separations. Their results show that in those cases the space-time correlations can be expressed in terms of space correlations. Corrsin [13] and Kovasznay [14] make a general conjecture that space-time correlations are the diminution of the space correlations by an unknown probability kernel. The choice of the kernel is ad hoc and crucial to the usefulness of the conjecture [15]. In this letter, we will develop an elliptic model for space-time correlations in turbulent shear flows. The model connects space-time correlations with space correlations via two characteristic velocities.

The present work is motivated by the fact that the spacetime correlations are important in turbulence statistical theory and subgrid scale (SGS) modeling for large eddy simulation (LES). In the closure theory of turbulence such as direct interaction approximation [2,11], EDQNM [10] and mapping closure approximation [16,17], the models for space-time correlations are needed. The recent application of LES to aeroacoustic suggests that the ability of LES to predict the space-time correlations will become increasingly important. The existing SGS models are, however, mostly constructed to predict spatial statistics such as energy spectra. It is not clear whether these SGS models can lead to the accu-

\footnotetext{
*Author to whom correspondence should be addressed. Email address: hgw@1nm.imech.ac.cn or guoweihe@yahoo.com
}

rate predictions of space-time spectra $[18,19]$. Hence, the relation between space-time correlations and space correlations is crucial to develop new SGS models for this purpose.

We consider the space-time correlations of streamwise velocity fluctuation $u_{1}$ in turbulent channel flows

$$
R\left(r, \tau ; x_{2}\right)=\left\langle u_{1}\left(x_{1}+r, x_{2}, x_{3}, t+\tau\right) u_{1}\left(x_{1}, x_{2}, x_{3}, t\right)\right\rangle,
$$

where $r$ is the spatially streamwise separation and $\tau$ the temporal separation. The ensemble average, denoted by bracket, is taken as the averaging over the $x_{1} x_{3}$ plane and a period of time $t$, since $u_{1}$ is homogeneous in the $x_{1} x_{3}$ plane and stationary in time. $R\left(r, \tau ; x_{2}\right)$ will be abbreviated as $R(r, \tau)$ without confusion.

A first approximation of space-time correlations by space correlations is known as Taylor's frozen flow hypothesis, or simply Taylor's hypothesis [20]: the spatial patterns of turbulent motion are carried past a fixed point by the convection speed $U$ without any essential changes. Hence, we have

$$
u_{1}\left(x_{1}+r, x_{2}, x_{3}, t+\tau\right)=u_{1}\left(x_{1}+r-U \tau, x_{2}, x_{3}, t\right) .
$$

Substitution of Eq. (2) into Eq. (1) yields

$$
R(r, \tau)=R(r-U \tau, 0),
$$

or, in Fourier space

$$
R(r, \tau)=\int E(k) \cos [k(r-U \tau)] d k,
$$

where $E(k)$ is the energy spectrum of the velocity $u_{1}$.

Equation (3) implies that the space-time correlations can be determined by space correlations and convection velocity. As a result, the correlations remain constant for all $r$ and $\tau$ along the straight lines $r-U \tau=c^{\prime}$ ( $c^{\prime}$ is constant). However, the result is inconsistent with the fact that the correlation functions decrease to zero as $r$ or $\tau$ goes to infinite. Therefore, Taylor's model (3) is not valid for large separations $r$ and $\tau$ in turbulent shear flows.

Several mechanisms [21-25] have been suggested by critically examining the validity of Taylor's hypothesis. Our above argument suggests an alternative explanation: Taylor's hypothesis implies that the iso-correlation contours are straight lines 


$$
r-U \tau=c^{\prime},
$$

where $c^{\prime}$ is a solution to Eq. (3): $R(r-U \tau, 0)=c$. However, both numerical simulations [26,27] and experiments [28] have shown that the iso-correlation contours in turbulent shear flows are the elongated and closed curves with a preference direction. They are not straight lines. Taylor's hypothesis implies a linear approximation to the contours, which is accurate only for highly elongated and very narrowed contours. Therefore, a higher order approximation to the isocorrelation contours has to be introduced [29].

We start with an iso-correlation contour $R(r, \tau)=c$. If we can find a point $\left(r_{c}, 0\right)$ on the contour, that is

$$
R(r, \tau)=R\left(r_{c}, 0\right),
$$

then the space-time correlations are determined by the space correlation $R(r, 0)$ and the solution $r_{c}$ of Eq. (6). It is shown in numerical simulations [26,27] and experiments [28] that the contours are closed curves with a single peak at the origin. This ensures the existence of a solution to Eq. (6).

Now we expand the correlation function $R(r, \tau)$ in the Taylor series about the origin up to second order

$$
\begin{aligned}
R(r, \tau)= & R(0,0)+\frac{\partial^{2} R(0,0)}{\partial r \partial \tau} r \tau \\
& +0.5\left[\frac{\partial^{2} R(0,0)}{\partial r^{2}} r^{2}+\frac{\partial^{2} R(0,0)}{\partial \tau^{2}} \tau^{2}\right],
\end{aligned}
$$

where $R_{r}^{\prime}(0,0)=0$ due to homogeneity and $R_{\tau}^{\prime}(0,0)=0$ due to stationary. Replacing $R$ in Eq. (6) by Eq. (7) yields

$$
r_{c}^{2}=(r-U \tau)^{2}+V^{2} \tau^{2}
$$

where

$$
\begin{gathered}
U=-\frac{\partial^{2} R(0,0)}{\partial r \partial \tau}\left[\frac{\partial^{2} R(0,0)}{\partial r^{2}}\right]^{-1}, \\
V^{2}=\frac{\partial^{2} R(0,0)}{\partial \tau^{2}}\left[\frac{\partial^{2} R(0,0)}{\partial r^{2}}\right]^{-1}-U^{2} .
\end{gathered}
$$

Substituting Eq. (8) into Eq. (6), we obtain the elliptic model

$$
R(r, \tau)=R\left[\sqrt{(r-U \tau)^{2}+V^{2} \tau^{2}}, 0\right],
$$

or, in Fourier space

$$
R(r, \tau)=\int E(k) \exp \left[i r k \sqrt{(r-U \tau)^{2}+V^{2} \tau^{2}}\right] d k
$$

The elliptic model is evidently valid for small separations $r$ and $\tau$, and it is also applicable for large separations $r$ and $\tau$. The applicability is supported by the similarity hypotheses: the iso-correlation contours share a preference direction and an aspect ratio, the latter is equal to the ratio of the decorrelation length scale in the fastest descent direction to the one in the slowest descent direction. In turbulent shear flows, the preference direction is mainly determined by the convection velocity $[27,28]$ and the aspect ratio determined by the turbulence intensity [21,22]. In the present work, the similarity hypotheses can be specialized as follows: the family of the elliptic contours shares a preference direction $\operatorname{tg} \alpha$ and an aspect ratio $\lambda$; the former is defined as the slope of the major axis and the latter defined as the length ratio of the major axis to the minor one:

$$
\begin{gathered}
\operatorname{tg}^{2} \alpha=4 U^{2} /\left[\sqrt{\left(1+U^{2}-V^{2}\right)^{2}+4 U^{2} V^{2}}+\left(1-U^{2}-V^{2}\right)\right]^{2} \\
\lambda^{2}=4 V^{2} /\left[\sqrt{\left(1+U^{2}-V^{2}\right)^{2}+4 U^{2} V^{2}}+\left(1+U^{2}+V^{2}\right)\right]^{2} .
\end{gathered}
$$

Therefore, the elliptic model implies that the iso-correlation contours are approximately represented by the elliptic curves

$$
(r-U \tau)^{2}+V^{2} \tau^{2}=c^{\prime} .
$$

If $V=0$, Eq. (13) is degenerated to the straight line Eq. (5), where the preference direction of Eq. (13) is coincident with the slope of Eq. (5) $(\operatorname{tg} \alpha=U)$ and the aspect ratio $\lambda$ becomes zero. It can be expected that the linear approximation holds for small $V$ and Taylor's hypothesis is thus valid. However, at larger $V$, the linear approximation is not accurate but the elliptic approximation is still applicable. On the other hand, if $U=0$, Eq. (13) becomes $r^{2}+V^{2} \tau^{2}=c^{\prime}$ with the preference direction in the horizontal axis. Kraichnan's sweeping hypothesis implies the same result as the elliptic model up to second order. In this sense, $V$ is associated with the sweeping velocity.

The parameters $U$ and $V$ in turbulent channel flows can be calculated directly from the Navier-Stokes equation, using Eq. (9)

$$
\begin{aligned}
U^{2}= & U_{1}^{2}+2 U_{1}\left[\frac{\partial U_{1}}{\partial x_{2}}\left\langle u_{2} \frac{\partial u_{1}}{\partial x_{1}}\right\rangle+\left\langle\frac{\partial u_{1}}{\partial x_{1}} u_{j} \frac{\partial u_{1}}{\partial x_{j}}\right\rangle\right] \\
& \times\left\langle\left(\frac{\partial u_{1}}{\partial x_{1}}\right)^{2}\right\rangle^{-1}+\cdots, \\
V^{2}= & 2 \frac{\partial U_{1}}{\partial x_{2}}\left[\left\langle u_{2} u_{j} \frac{\partial u_{1}}{\partial x_{j}}\right\rangle\left\langle\left(\frac{\partial u_{1}}{\partial x_{1}}\right)^{2}\right\rangle^{-1}\right. \\
& -\left\langle u_{2} \frac{\partial u_{1}}{\partial x_{1}}\right\rangle\left\langle u_{j} \frac{\partial u_{1}}{\partial x_{1}} \frac{\partial u_{1}}{\partial x_{j}}\right\rangle\left\langle\left(\frac{\partial u_{1}}{\partial x_{1}}\right)^{2}\right\rangle-2 \\
& +\left\langle\left(u_{j} \frac{\partial u_{1}}{\partial x_{j}}\right)^{2}\right\rangle\left\langle\left(\frac{\partial u_{1}}{\partial x_{1}}\right)^{2}\right\rangle-1 \\
& \left.-\left\langle\frac{\partial u_{1}}{\partial x_{1}} u_{j} \frac{\partial u_{1}}{\partial x_{j}}\right\rangle^{2}\left\langle\left(\frac{\partial u_{1}}{\partial x_{1}}\right)^{2}\right\rangle-2\right]+\cdots,
\end{aligned}
$$

where $U_{1}$ is the mean streamwise velocity. The dots represent the higher-order terms associated with the velocity fluctuations and the mean shear rates. Note that the higher-order terms do not contain the mean velocity $U_{1}$. If $U_{1}$ is dominating and the velocity fluctuations and the shear rates are relatively smaller, then $U$ is dominating and $V$ could be ignored. In this case, Taylor's hypothesis is valid; If $U_{1}$ is zero and $u_{j}$ isotropy, we can use the quasi-normal assumption to calculate $U=0$ and $V^{2}=\left\langle u_{j} u_{j}\right\rangle$. This is the case for Kraichnan's sweeping hypothesis, where $V$ represents a sweeping velocity. These arguments justify Lin and Lumley's criteria 


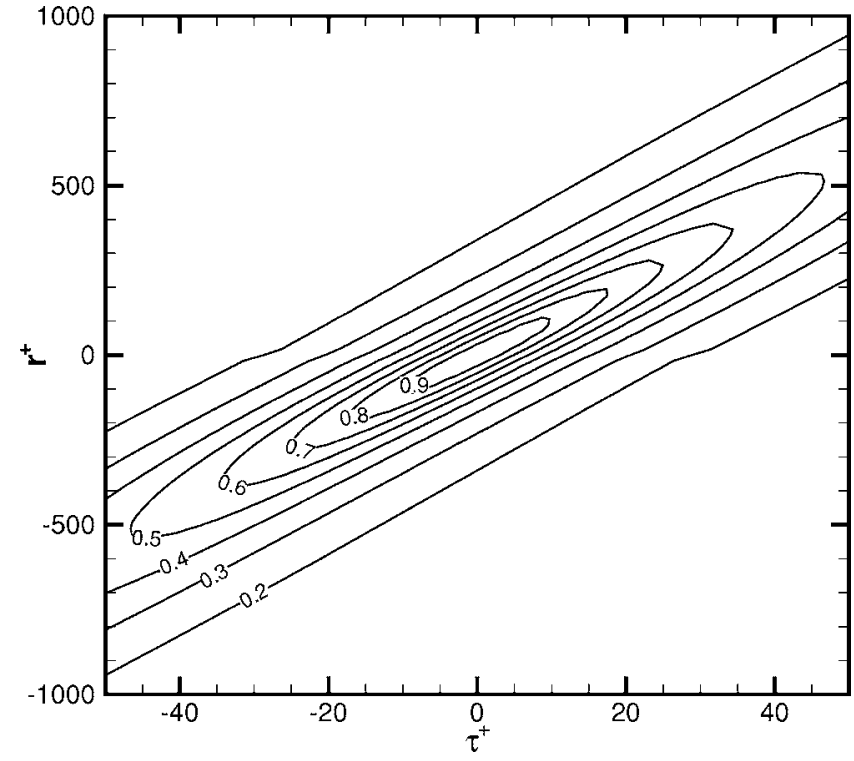

FIG. 1. Contours of space-time correlations $R\left(r, \tau ; x_{2}^{+}\right)$at $x_{2}^{+}$ $=12$ as a function of space and time separations.

[21,22] for Taylor's hypothesis: low intensity, weak shear rates and isotropy.

The performance of the elliptic model can be assessed by the data from direct numerical simulation (DNS) of turbulent channel flows. The DNS was performed using the standard spectral method [30]. The necessary parameters for DNS are as follows: $\operatorname{Re}_{\tau}=u_{\tau} \delta / \mu=180$ where $u_{\tau}$ and $\delta$ are the friction velocity and channel half-width, respectively; the computational domain is $4 \pi \delta, 2 \delta$ and $2 \pi \delta$ for the streamwise, normal and spanwise directions, respectively, and the grid resolution is $128 \times 129 \times 128$.

Figure 1 plots the contours of space-time correlations, $R(r, \tau)=c$, at $x_{2}^{+}=12$. These contours are very different from

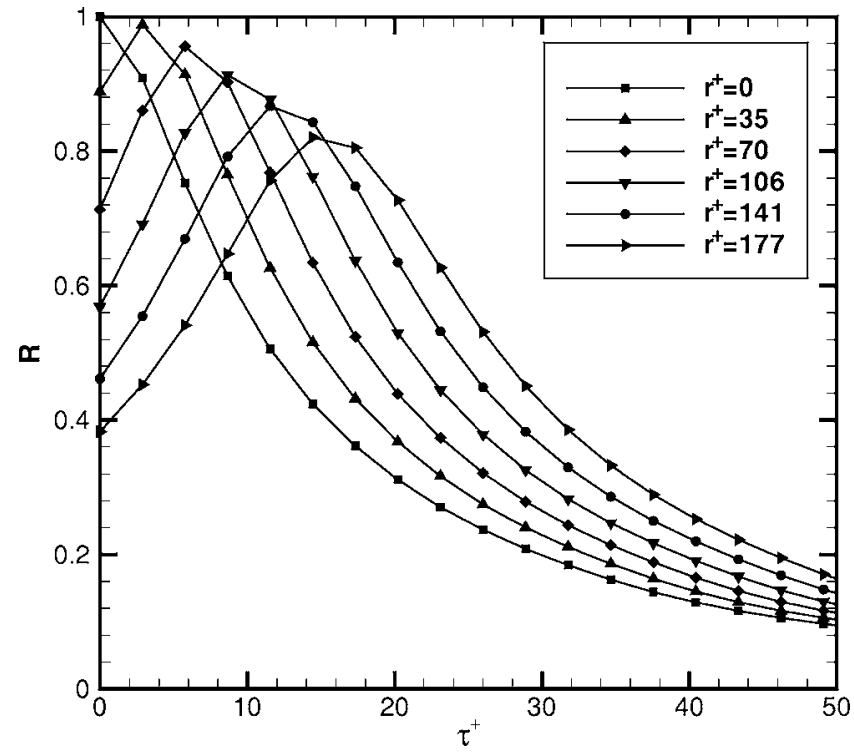

FIG. 2. Space-time correlations $R\left(r, \tau ; x_{2}^{+}\right)$for different space separations $r^{+}=0,35,70,106,141,177$ at $x_{2}^{+}=12$ vs time separation $\tau$.

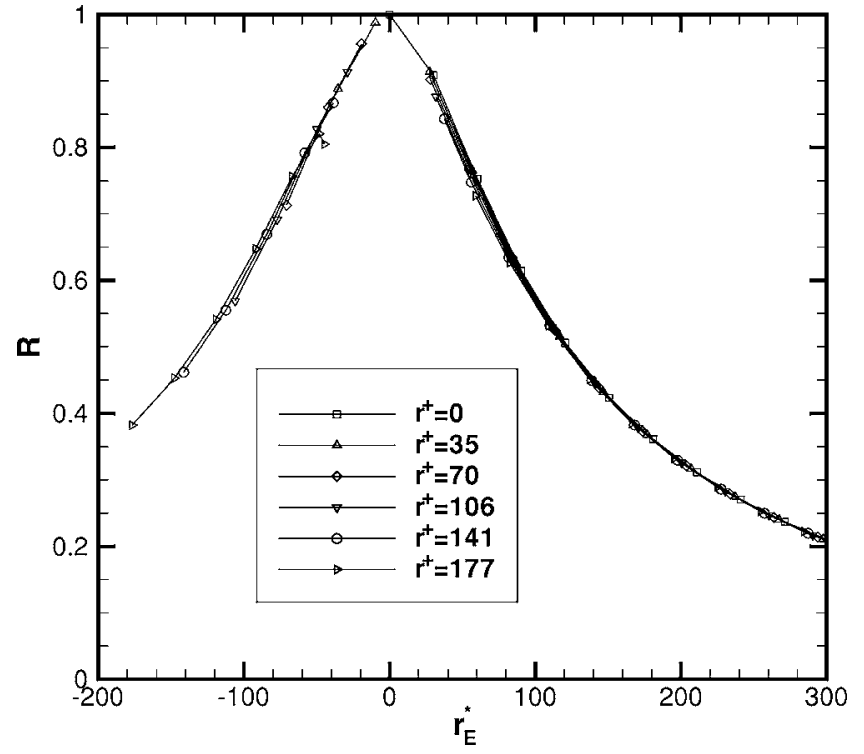

FIG. 3. Space-time correlations $R\left(r, \tau ; x_{2}^{+}\right)$for different space separations $r$ at $x_{2}^{+}=12$ vs the separation $r_{E}^{*}=\sqrt{(r-U \tau)^{2}+V^{2} \tau^{2}}$ defined by the elliptic model.

the straight lines as explained in Taylor's hypothesis. They are more like the elliptic curves in alignment with a straight line of the slope $U$. Figure 2 shows the evolution of the space-time correlations $R$ with respective to time $\tau$ for different space separations $r^{+}=0,35,70,106,141,177$ at the location $x_{2}^{+}=12$. These correlation curves initially raise to the maximums and then decay.

In Fig. 3, these results are all plotted together with the separation axis defined by the two-velocity-dependent variable $r_{E}^{+}= \pm \sqrt{\left(r^{+}-U \tau^{+}\right)^{2}+V^{2}\left(\tau^{+}\right)^{2}}$. Here the parameters $U$ and $V$ are calculated from the DNS data using Eq. (12), where $\operatorname{tg} \alpha$ is the mean preference directions and $\lambda$ the averaged

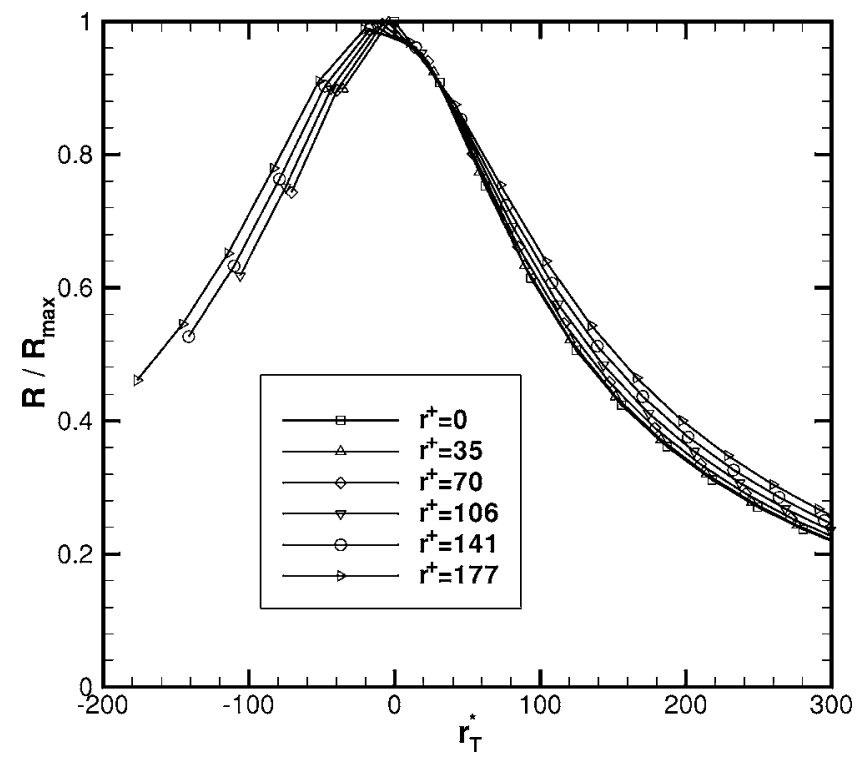

FIG. 4. The normalized space-time correlations $R\left(r, \tau ; x_{2}^{+}\right) / R_{\max }$ for different space separations $r$ at $x_{2}^{+}=12$ vs the separation $r_{T}^{*}=r$ $-U \tau$ defined by Taylor's hypothesis. 
aspect ratio, $\tau^{+}$is positive for $\tau \geq \tau_{0}$ and $\tau^{+}$is negative for $\tau<\tau_{0}$, where $\tau_{0}$ maximizes the correlation $R\left(r_{0}, \tau\right)$ for a given separation $r_{0}$. This normalization causes an excellent collapse of the correlation curves. We have also used Taylor's hypothesis to plot the space-time correlations, normalized by their maximums, against the convection-velocitydependent variable $r_{T}^{+}=r^{+}-U \tau^{+}$, see Fig. 4. The final outcome is not as good as that for the elliptic model.

The space-time correlations for other $x_{2}$ locations, such as $x_{2}^{+}=5$ and $x_{2}^{+}=149$, were also plotted in the same ways as ones in Figs. 1-4. These results exhibit the same behaviors as predicted by the elliptic model. For $x_{2}^{+}=5,12$ and 149 , $(U, V)$ are calculated as $(9.8,3.58),(10.1,2.58)$ and $(17.3,0.9)$, respectively. As expected, the convection velocity $U$ increases with $x_{2}^{+}$increasing and the sweeping velocity $V$ decreases with $x_{2}^{+}$increasing.

The elliptic model is developed upon a second-order approximation to the iso-correlation contours. Successively higher-order approximation can be achieved, if necessary, by including the higher-order terms of the Taylor series of the correlation functions. Formally, the contour functions can be expanded in a Taylor series and the Taylor coefficients can thus be calculated from the Navier-Stokes equations. The truncated Taylor series up to a higher-order offers more information on the relationship between space-time correlations and space correlations.

In summary, we develop an elliptic model for space-time correlations in turbulent shear flows. This model relates the space-time correlations to the space correlations via the convection velocity and the sweeping velocity. The convection velocity is mainly determined by the local mean velocity and the sweeping velocity determined by the turbulent intensity and shear rates. The data from the DNS of turbulent channel flows demonstrate that the elliptic model is valid in the sense that the normalized correlation curves in terms of the elliptic model almost collapse.

\section{ACKNOWLEDGMENTS}

G.-W. H. thanks Dr. Zhongmin Xiong for his constantly stimulating discussions and Professor Tianshu Liu for his comments on the manuscript. This work was supported by Chinese Academy of Sciences under the innovative project "Multi-scale modeling and simulation in complex system" and National Natural Science Foundation of China under the project No. 10325211.
[1] G. L. Eyink, Phys. Rev. E 62, 210 (2000).

[2] R. H. Kraichnan, Phys. Fluids 7, 1723 (1964).

[3] G. Comte-Bellot and S. Corrsin, J. Fluid Mech. 48, 273 (1971).

[4] H. Tennekes, J. Fluid Mech. 67, 561 (1975).

[5] Y. Kaneda and T. Gotoh, Phys. Fluids A 3, 1924 (1991).

[6] R. Rubinstein and Y. Zhou, Phys. Fluids 11, 2288 (2000).

[7] M. Quadrio and P. Luchini, Phys. Fluids 15, 2219 (2003).

[8] R. Camussi and R. Verzicco, Eur. J. Mech. B/Fluids 23, 427 (2004).

[9] A. Jachens, J. Schumacher, B. Eckhardt, K. Knobloch, and H. Z. Fernholz, J. Fluid Mech. 547, 55 (2006).

[10] U. Frisch, Turbulence (Cambridge University Press, Cambridge, 1995).

[11] D. C. Leslie, Modern Development in the Theory of Turbulence (Oxford University Press, Oxford, 1972).

[12] T. Gotoh and Y. Kaneda, Phys. Fluids A 3, 2426 (1991).

[13] S. Corrsin, Proceeding of the Oxford Symposium on Atmospheric Diffusion and Air Pollution (Academic, New York, 1960), p. 162.

[14] L. S. G. Kovasznay, J. Aeronaut. Sci. 20, 657 (1953).

[15] W. R. C. Phillips, Phys. Fluids 12, 2056 (2000).
[16] H. Chen, S. Chen, and R. H. Kraichnan, Phys. Rev. Lett. 63, 2657 (1989).

[17] G.-W. He and Z. F. Zhang, Phys. Rev. E 70, 036309 (2004).

[18] G.-W. He, R. Rubinstein, and L. P. Wang, Phys. Fluids 14, 2186 (2002).

[19] M. Wang, J. B. Freund, and S. K. Lele, Annu. Rev. Fluid Mech. 38, 483 (2006).

[20] G. I. Taylor, Proc. R. Soc. London, Ser. A 164, 476 (1938).

[21] C. C. Lin, Q. Appl. Math. 10, 295 (1953).

[22] J. L. Lumley, Phys. Fluids 8, 1056 (1965).

[23] U. Piomelli, J.-L. Ballint, and J. M. Wallace, Phys. Fluids A 1, 609 (1989).

[24] C. Tong and Z. Warhaft, J. Fluid Mech. 292, 1 (1995).

[25] T. Burghelea, E. Segre, and V. Steinberg, Phys. Fluids 17, 103101 (2005).

[26] H. Choi and P. Moin, Phys. Fluids A 2, 1450 (1990).

[27] J. Kim and F. Hussain, Phys. Fluids A 5, 695 (1993).

[28] J. A. B. Wills, J. Fluid Mech. 20, 417 (1964).

[29] G. W. He and M. Wang, Annual Research Briefs 2004, pp. 195-199, Stanford University.

[30] J. Kim, P. Moin, and R. Moser, J. Fluid Mech. 177, 133 (1987). 